\title{
Using an Improved Artificial Bee Colony Algorithm for Parameter Estimation of a Dynamic Grain Flow Model
}

\author{
He Wang $(\mathbb{D})^{1}$ Hongbin Liang, ${ }^{1}$ and Lei Gao ${ }^{2}{ }^{2}$ \\ ${ }^{1}$ School of Mechanical Engineering and Automation, University of Science and Technology Liaoning, Anshan 114051, China \\ ${ }^{2}$ Department of Information Service and Intelligent Control, Shenyang Institute of Automation, Chinese Academy of Sciences, \\ Shenyang 110016, China
}

Correspondence should be addressed to He Wang; wanghe@ustl.edu.cn

Received 31 July 2017; Revised 9 November 2017; Accepted 4 January 2018; Published 31 January 2018

Academic Editor: Stefan Balint

Copyright (C) 2018 He Wang et al. This is an open access article distributed under the Creative Commons Attribution License, which permits unrestricted use, distribution, and reproduction in any medium, provided the original work is properly cited.

\begin{abstract}
An effective method is proposed to estimate the parameters of a dynamic grain flow model (DGFM). To this end, an improved artificial bee colony (IABC) algorithm is used to estimate unknown parameters of DGFM with minimizing a given objective function. A comparative study of the performance of the IABC algorithm and the other $A B C$ variants on several benchmark functions is carried out, and the results present a significant improvement in performance over the other $A B C$ variants. The practical application performance of the IABC is compared to that of the nonlinear least squares (NLS), particle swarm optimization (PSO), and genetic algorithm (GA). The compared results demonstrate that IABC algorithm is more accurate and effective for the parameter estimation of DGFM than the other algorithms.
\end{abstract}

\section{Introduction}

Grain flow sensor is an important part of yield monitoring system in precision agriculture. There are mainly four types of grain flow sensor: impact-type [1], ray-type [2], volume-type [3], and weight-type [4]. Most commercial yield monitoring systems rely on impact-type for security, reliability, and simple structure. Impact-type measures the grain force exerted on the sensing device usually at the exit of the clean grain elevator, shown in Figure 1. The measured force is related to the grain flow.

Grain flow model is used to characterize the relationship between the grain flow and the impact force, which contains linear models and nonlinear models. Linear models have been utilized due to being easy for model building [5-8]. The results of the experiment, by contrast, denote a strongly nonlinear relation between the grain flow and the impact force at larger grain flow [9], so nonlinear models have been proposed for grain flow sensor to estimate a wide range of grain flow $[10,11]$ and are gradually employed for mass flow sensors to minimize the effects of changes in material properties $[12,13]$. At this time, nonlinear regression methods are used to estimate parameter of nonlinear models with minimizing a given objective function [11, 14, 15]. But these methods have disadvantages of low convergence precision and slow convergence rate.

Recently, many artificial intelligent techniques have emerged as useful tool to solve complex problems, and these techniques have been utilized to parameter estimation of nonlinear models. In [16], application of an ant colony optimization (ACO) algorithm on estimation of the ultrasonic echo parameters shows the feasibility of ACO algorithm. The artificial bee colony algorithm $(\mathrm{ABC})$ is used to search the appropriate combinations of the driving waveform parameters [17]. The particle swarm optimization (PSO) to determine the unknown photovoltaic (PV) model parameters was proposed by Soon and Low [18]. A multiobjective genetic algorithm (MOGA) is utilized to determine reliable estimates of the physical parameters of a complex robotic arm which are not known in advance [19]. Artificial intelligence techniques are superior to the traditional methods and have the obvious advantages of solving the global optimization problem with high precision and short time. However, artificial intelligent techniques still have some challenges to overcome, such 


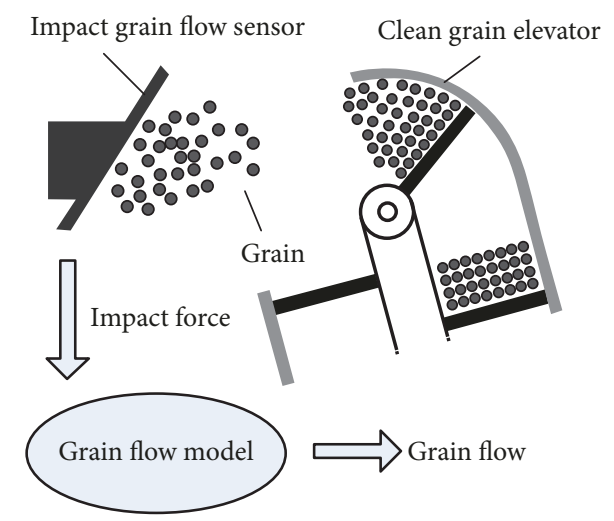

FIGURE 1: Work schematic of impact grain flow sensor.

as problem of falling into local optimums and problem of convergence rate.

$\mathrm{ABC}$ has been proven to be a powerful and efficient tool for real-world optimization problems [20-22]. In order to efficiently and accurately estimate parameters of nonlinear grain flow models, this paper is to develop an improved artificial bee colony (IABC) algorithm and employ IABC to estimate parameter of DGFM and compare the results with other methods. Therefore, we have redefined new food sources in original $\mathrm{ABC}$ algorithm to obtain a new variant version of this algorithm which, compared to the other $A B C$ variants, is highly effective for improved search performance.

The paper is organized as follows. In Section 2, related works about DGFM and ABC algorithm are introduced. In Section 3, IABC algorithm is detailed. In Section 4, IABC algorithm is compared with the other $\mathrm{ABC}$ variants on benchmark functions. In Section 5, IABC algorithm is used to estimate parameters of DGFM and comparison results are presented. Conclusions are made in Section 6.

\section{Relate Works}

2.1. DGFM. As mentioned above, it is a trend to model grain flow using nonlinear models. DGFM is proposed to give an accurate estimation of grain flow [23]. DGFM is classified into four stages: the filling stage, the settling stage, the release stage, and the flight stage.

2.1.1. Filling Stage. Total grain mass on a paddle is obtained as follows:

$$
M=\dot{m} \int P(t) d t=\dot{m} \frac{d}{V},
$$

where $M$ is total grain mass on a paddle, $\dot{m}$ is the mass flow, $P(t)$ is the coefficient of opening of the auger at time $t, d$ is the distance between two paddles, and $V$ is the linear velocity of the paddles.

2.1.2. Settling Stage. $h(\psi)$ is the distance between the sprocket center and inner boundary of the grains at any angle. $\psi$ is calculated as follows:

$$
h(\psi)=l\left(\frac{l}{h_{0}} \cos \psi+\left(1-\frac{l}{h_{0}} \cos \psi_{f}\right) \frac{\sin \psi}{\sin \psi_{f}}\right)^{-1},
$$

where $l$ is length of the paddle and $\psi_{f}$ is the maximal angle achieved by the grain distribution. Specifically, $h(0)=h_{0}$, $h\left(\psi_{f}\right)=l . \psi_{f}$ and $h_{0}$ can be obtained from (3) and (4), respectively.

$$
\begin{aligned}
\psi_{f} & =\frac{h_{f}\left(l-h_{0}\right)}{l} \\
\frac{M}{\eta \omega l^{2}} & =\frac{h_{f}\left(l-h_{0}\right)-h_{0} \sin \left(h_{f}\left(\left(l-h_{0}\right) / l\right)\right)}{2 l},
\end{aligned}
$$

where $h_{f}$ is the grain shape coefficient to be estimated, $\eta$ is the density of the grain, and $w$ is the width of the paddle.

2.1.3. Release Stage. Horizontal velocity and vertical velocity at the end of the release stage are given by

$$
\begin{gathered}
v_{x}=l \Omega\left(\widetilde{\rho}^{\prime}\left(\tau_{d}\right) \cos \left(\theta_{d}\right)-\sin \left(\theta_{d}\right)\right) \\
v_{y}=l \Omega\left(\widetilde{\rho}^{\prime}\left(\tau_{d}\right) \cos \left(\theta_{d}\right)+\sin \left(\theta_{d}\right)\right) \\
\tilde{\rho}^{\prime}\left(\tau_{d}\right)=\frac{1}{2}\left(\widetilde{\rho}_{0}-\frac{\widetilde{\rho}_{0} \mu}{\sqrt{1+\mu^{2}}}\right) \\
\cdot e^{\left(-\mu-\sqrt{1+\mu^{2}}\right) \tau_{d}}\left(-\mu-\sqrt{1+\mu^{2}}\right) \\
+\frac{1}{2}\left(\widetilde{\rho}_{0}+\frac{\widetilde{\rho}_{0} \mu}{\sqrt{1+\mu^{2}}}\right) \\
\cdot e^{\left(-\mu+\sqrt{1+\mu^{2}}\right) \tau_{d}}\left(-\mu+\sqrt{1+\mu^{2}}\right) \\
\theta_{d}=\frac{3 \pi}{2}+\Omega \tau_{d},
\end{gathered}
$$

where $v_{x}$ is the horizontal velocity, $v_{y}$ is the vertical velocity, $\Omega$ is the sprocket rotational speed, $\mu$ is the coefficient of friction, $\widetilde{\rho}_{0}$ is coefficient of radial distance, and $\tau_{d}$ is the travel time of grain and can be obtained from

$$
\begin{aligned}
1= & \frac{1}{2}\left(\tilde{\rho}_{0}-\frac{\tilde{\rho}_{0} \mu}{\sqrt{1+\mu^{2}}}\right) e^{\left(-\mu-\sqrt{1+\mu^{2}}\right) \tau_{d}} \\
& +\frac{1}{2}\left(\tilde{\rho}_{0}+\frac{\tilde{\rho}_{0} \mu}{\sqrt{1+\mu^{2}}}\right) e^{\left(-\mu+\sqrt{1+\mu^{2}}\right) \tau_{d}} .
\end{aligned}
$$

2.1.4. Flight Stage. The impact force caused by grain can be expressed as 


$$
F= \begin{cases}\frac{V}{d} \int_{\rho_{\text {cit }}}^{1}\left(v_{x} \sin \phi-v_{y} \cos \phi\right) \sin \phi(1+e) \rho h^{-1}(\rho) \eta w d \rho & \rho_{\text {crit }}>h_{0} \\ \frac{V}{d} \int_{h_{0}}^{1}\left(v_{x} \sin \phi-v_{y} \cos \phi\right) \sin \phi(1+e) \rho h^{-1}(\rho) \eta w d \rho & h_{0}>\rho_{\text {crit }}\end{cases}
$$

where $F$ is impact force, $\phi$ is installation angle of grain flow sensor, $e$ is the coefficient of restitution, $h^{-1}(\rho)$ is used to compute the opening angle $\psi$, and $h(\psi)=\rho \rho_{\text {crit }}$ is the critical radius, which meet the condition: $\lambda>0, \kappa=0 . \lambda$ and $\kappa$ are calculated as follows:

$$
\begin{aligned}
& \lambda=\frac{\left(d_{x}-l \cos \theta_{d}\right) \sin \phi-\left(d_{y}-l \sin \theta_{d}\right) \cos \phi}{v_{x} \sin \phi-v_{y} \sin \phi}, \\
& \kappa=\frac{v_{x}\left(d_{y}-l \sin \theta_{d}\right)-v_{y}\left(d_{x}-l \cos \theta_{d}\right)}{v_{y} \cos \phi-v_{x} \sin \phi},
\end{aligned}
$$

where $d_{x}$ is the horizontal distance between the lowest point of grain flow sensor and sprocket center and $d_{y}$ is the vertical distance between the lowest point of grain flow sensor and sprocket center.

DGFM is represented by (7), which describes a physical relationship between the impact force and the grain flow with multiple parameters. The simplified form of the model is as follows:

$$
F=f\left(\dot{m}, \mu, e, \eta, h_{f}, V, d, \Omega, l, w, d_{x}, d_{y}, \phi\right) .
$$

In (9), as discussed above, the eight parameters $\left[\begin{array}{llllllll}V & d & \Omega & l & w & d_{x} & d_{y} & \phi\end{array}\right]$ are the machine geometric parameters obtained by measurement; the four parameters $\left[\begin{array}{llll}\mu & e & \eta & h_{f}\end{array}\right]$ are theoretical parameters to be estimated, and $f(*)$ is the value calculated with DGFM. Mass flow is calculated by applying a bisection algorithm to DGFM. Establishment and application of the DGFM can be found in the literature [23].

2.2. Objective Function. As discussed above, the DGFM consists of four unknown parameters. Unknown parameter estimation problem can be transformed into an optimization problem by minimizing a root mean square error (RMSE). For this purpose, the impact force is measured and computed by grain flow sensor and DGFM, respectively. Unknown parameters are estimated by the proposed objective function defined as follows:

$$
\mathrm{RMSE}=\sqrt{\frac{1}{M} \sum_{i=1}^{M}\left(F_{i}-F_{i}^{\prime}\right)^{2}},
$$

where $M$ is the number of samples, $F_{i}$ is the $i$ th calculated impact force in terms of (9), and $F_{i}^{\prime}$ is the $i$ th measured impact force.

2.3. Review of $A B C$ Algorithm. The $A B C$ algorithm simulating the foraging process of honey bees was proposed to solve complex optimization problems [24]. ABC algorithm contains three types of bees: employed bees, onlooker bees, and scout bees. Three types of bees share the information of the food sources with each other. Employed bees find the food sources and deliver the information of the food sources to onlooker bees in the hive. Onlooker bees follow employed bees to explore the food sources based on the information they have received. In the process of looking for the food sources, if the food source is not improved after a continuous trial at predetermined value, then employed bee abandon that food sources and turn into a scout searching around randomly. Predetermined value for abandoning a food source is called "limit," which is an important control parameter in the $\mathrm{ABC}$ algorithm. The flow of the $\mathrm{ABC}$ algorithm is listed as follows.

Step 1. $N$ food sources are generated randomly from the following:

$$
x_{i j}=x_{j}^{\min }+\operatorname{rand}[0,1]\left(x_{j}^{\max }-x_{j}^{\min }\right),
$$

where $x_{i j}$ represents that artificial bee is at the $j$ th position of the $i$ th food source; $i=1,2, \ldots, N ; j=\operatorname{rand}[1,2, \ldots, n] ; N$ is the number of food sources; $n$ is the search space dimension; $x_{j}^{\min }$ and $x_{j}^{\max }$ are the upper and lower limits of the $j$ th variable, respectively.

Step 2. If the current food position is $X_{i}=\left[x_{i 1}, x_{i 2}, \ldots, x_{i n}\right]$, the following solution search equation is used by the employed bees to generate a new food source $V_{i}=$ $\left[x_{i 1}, x_{i 2}, \ldots, v_{i j}, \ldots, x_{i n}\right]$.

$$
v_{i j}=x_{i j}+\theta_{i j}\left(x_{i j}-x_{k j}\right),
$$

where $v_{i j}$ represents the position of new source found by the $i$ th employed bee, $i=1,2, \ldots, N, j=\operatorname{rand}[1,2, \ldots, n], k \in$ $\{1,2, \ldots, N\}$, and $k \neq i ; \theta_{i j}$ is a random number selected from $[-1,1]$.

Step 3. Each onlooker bee chooses and follows the employed bees based on the quality of food sources. The quality of food sources denoted as $P_{i}$ is given by

$$
P_{i}=\frac{\text { fit }_{i}}{\sum_{i=1}^{N} \mathrm{fit}_{i}},
$$

where $\mathrm{fit}_{i}$ is the fitness value of the $i$ th solution. For a minimization problem, the fitness value of a solution can be defined as

$$
\text { fit }_{i}= \begin{cases}\frac{1}{1+f\left(X_{i}\right)} & \text { if } f\left(X_{i}\right)>0 \\ 1+\left|f\left(X_{i}\right)\right| & \text { if } f\left(X_{i}\right) \leq 0\end{cases}
$$

where $f\left(X_{i}\right)$ is the value of the objective function at $X_{i}$. 
Step 4. If a food source fails to get better after predetermined value "limit," then that food source needs to be abandoned. The employed bees become scouts later. A new food source is randomly generated in space using (11).

\section{IABC Algorithm}

This section presents the detailed description of the proposed improved artificial bee colony (IABC) algorithm. There are two improvements on basic ABC algorithm: modifying the search method of the onlooker bees and proposing a novel probability model.

3.1. Search Method for the Onlooker Bees. In ABC, production of new food source (see in (12)) reduces the effectiveness of algorithm because existing information is not used to produce new food source. Inspired by differential evolution (DE), Zhu and Kwong [25], proposed a modified ABC algorithm (MABC) which uses information of global best food source. Search method of MABC can be expressed as follows:

$$
v_{i j}=x_{\text {best }, j}+\theta_{i j}\left(x_{r_{1}, j}-x_{r_{2}, j}\right),
$$

where $X_{\text {best }}$ is the best food source in the all food source. The indices $r_{1}$ and $r_{2}$ are mutually exclusive integers randomly chosen from $\{1,2, \ldots, N\}$ and different from the base index $i$.

In IABC, the search method of MABC is modified to further improve the exploitation. The new method used to calculate a new food source is shown in

$$
v_{i j}=x_{\text {best }, j}+\theta_{i j}\left(x_{\text {best }, j}-x_{r_{2}, j}\right) .
$$

Compared with (15), (16) not only produces the new food source around the best food source but also makes use of the information of the best food source to guide the search trajectory. Thus, the exploitation of algorithm is improved greatly.

3.2. Probability Model. It is obvious that (16) has a good capability of exploitation. As a result, the diversity of population decreases. In IABC, hence, a probability model is used to increase the diversity of population, and it can be expressed as follows:

$$
P_{i}=0.8 \times \frac{\mathrm{fit}_{i}}{\mathrm{fit}_{\max }}+0.2,
$$

where fit $_{\max }$ is the maximum fitness value of population.

In (17), values 0.8 and 0.2 are the optimal through simulation test and ensure some diversity in the case when one solution is superior to all others by allowing $20 \%$ of onlooker bees to select different solution.

From the above analysis, two equations are modified ((12) and (13) are replaced with (16) and (17), resp.), but the main framework of IABC is consistent with that of ABC.

\section{Simulation Results and Analysis}

A set of six benchmark functions is applied to verify the performance of IABC in the simulation experiments. The benchmark functions are summarized in Table 1.
TABLE 1: Detail for the 6 benchmark functions used in experiments.

\begin{tabular}{lccc}
\hline Functions & Dimension & Search range & Optimum value \\
\hline Rosenbrock & 2 and 3 & {$[-30,30]$} & 0 \\
Schaffer & 2 and 3 & {$[-100,100]$} & 0 \\
Rastrigin & 30 and 60 & {$[-5.12,5.12]$} & 0 \\
Griewank & 30 and 60 & {$[-600,600]$} & 0 \\
Sphere & 30 and 60 & {$[-100,100]$} & 0 \\
Ackley & 30 and 60 & {$[-32,32]$} & 0 \\
\hline
\end{tabular}

Three ABC variants, GABC [25], MABC [26], and EABC [27], are used to compare with IABC. To be fair, the population size is set to 80 that are the same as that in [25]. To investigate the effect of limit, a test is made on four 30-dimensional test functions (Sphere, Griewank, Rastrigin, and Ackley) with IABC and the results are shown in Table 2. As seen from Table 2, limit can influence the optimization results. For the Griewank function, a faster convergence speed and better results are obtained, when limit is 200 . For the other three test functions, the effect of limit on the performance of IABC is very little except Rastrigin at limit $=400$. Therefore, in our experiments, the limit is set to 200 for all test functions.

Given the above, the population size, the maximum number of generations, and the limit values are set to 80 , 5000 , and 200, respectively. To be specific, the constant value $C$ is equal to 1.5 in GABC and the selective probability $P$ is set to 0.7 in MABC. Each of the experiments is repeated 30 times independently. The final results are given in Table 3.

Table 3 clearly shows that IABC works better than $\mathrm{ABC}$, $\mathrm{GABC}, \mathrm{EABC}$, and MABC. Although the result of IABC is inferior to that of $\mathrm{MABC}$ on the Rosenbrock, there is little difference between $\mathrm{MABC}$ and IABC. And it is worth noting that IABC maintains the framework of original $A B C$ and does not use chaotic maps and opposition-based learning techniques that are used in the initialization step of MABC.

Figure 2 presents the convergence curves of $\mathrm{ABC}, \mathrm{GABC}$, $M A B C$, and IABC on six benchmark functions with different dimension. From Figure 2, accuracy and convergence rate of IABC has been greatly improved compared to ABC, GABC, and $\mathrm{MABC}$ algorithm.

\section{Parameter Estimation of Dynamic Grain Flow Model with IABC}

To make it more accurate for DGFM to calculate grain flow, it is very necessary to use a more efficient optimization algorithm to solve parameters estimation problem of DGFM. In view of the excellent performance of the proposed IABC algorithm in this paper, the IABC algorithm is used for parameters estimation of DGFM. A schematic diagram of the approach is illustrated in Figure 3.

Impact force and grain flow signals were obtained from a test rig illustrated in Figure 4. The experiment conditions are as follows: a novel grain flow sensor was installed at the top of the clean grain elevator, using PVDF piezoelectric film as the active element to measure the impact force [28, 29]. Grain flow was measured by weighing sensor with accuracy of 

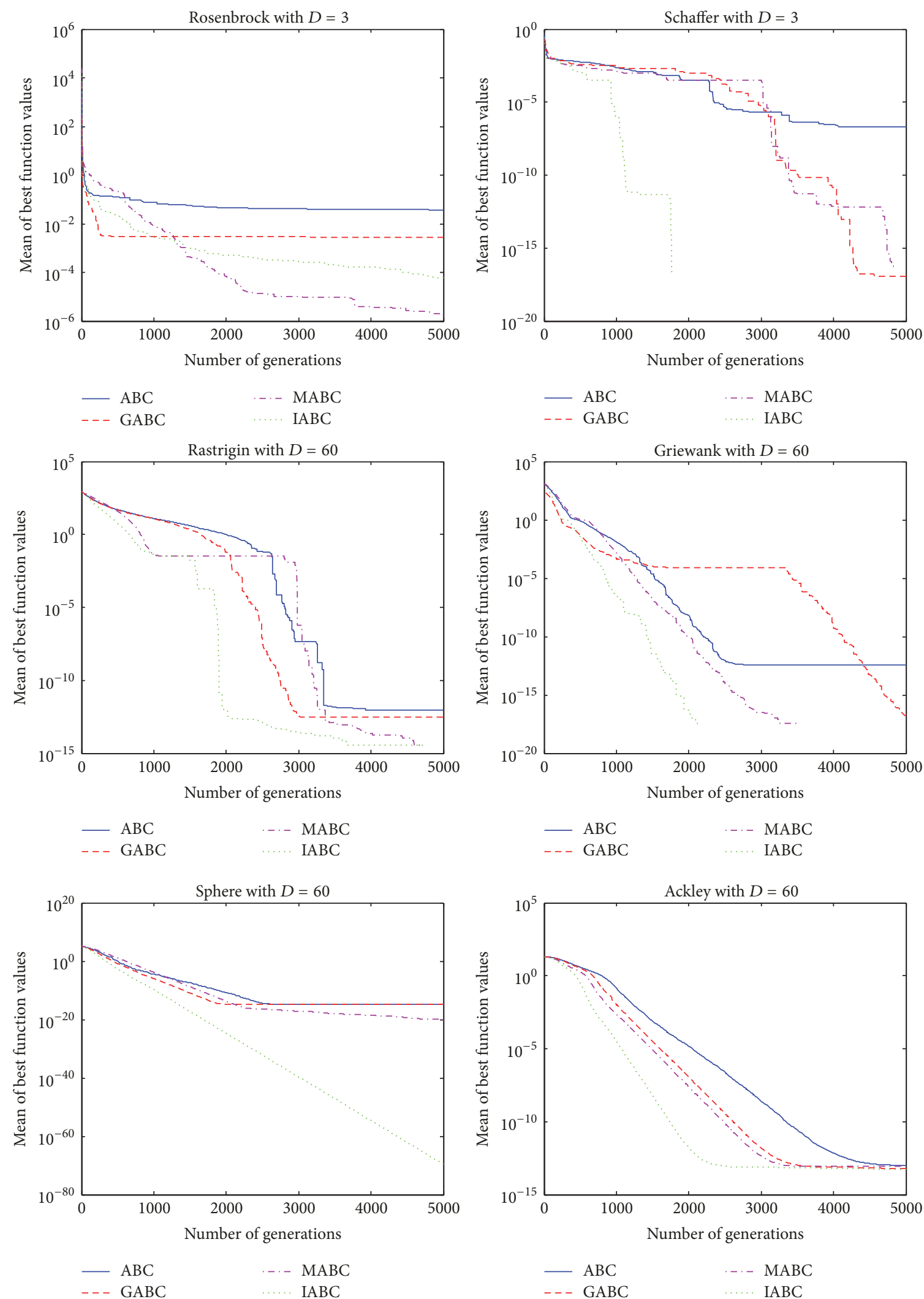

FIgURE 2: Convergence performance of $\mathrm{ABC}, \mathrm{GABC}, \mathrm{MABC}$, and IABC on six benchmark functions. 
TABLE 2: Effect of limit on the performance of IABC.

\begin{tabular}{|c|c|c|c|c|c|c|c|}
\hline \multirow{2}{*}{ Maximum number of generations } & \multirow{2}{*}{ Dimension } & \multirow{2}{*}{ Limit } & & \multicolumn{4}{|c|}{ Functions } \\
\hline & & & & Rastrigin & Griewank & Sphere & Ackley \\
\hline \multirow{2}{*}{1000} & \multirow{2}{*}{30} & \multirow{2}{*}{100} & Mean & 0 & $7.24 e-11$ & $5.58 e-27$ & $1.42 e-11$ \\
\hline & & & Std & 0 & $2.65 e-11$ & $7.41 e-27$ & $5.75 e-12$ \\
\hline \multirow{2}{*}{1000} & \multirow{2}{*}{30} & \multirow{2}{*}{200} & Mean & 0 & $2.96 e-12$ & $1.32 e-27$ & $1.26 e-13$ \\
\hline & & & Std & 0 & $4.99 e-12$ & $2.65 e-28$ & $3.48 e-14$ \\
\hline \multirow{2}{*}{1000} & \multirow{2}{*}{30} & \multirow{2}{*}{300} & Mean & 0 & $2.36 e-11$ & $2.95 e-27$ & $1.28 e-13$ \\
\hline & & & Std & 0 & $4.99 e-11$ & $3.19 e-27$ & $2.66 e-14$ \\
\hline \multirow{2}{*}{1000} & \multirow{2}{*}{30} & \multirow{2}{*}{400} & Mean & $4.64 e-5$ & $2.33 e-10$ & $1.72 e-26$ & $1.33 e-13$ \\
\hline & & & Std & $1.93 e-5$ & $3.15 e-10$ & $2.84 e-26$ & $3.69 e-14$ \\
\hline
\end{tabular}

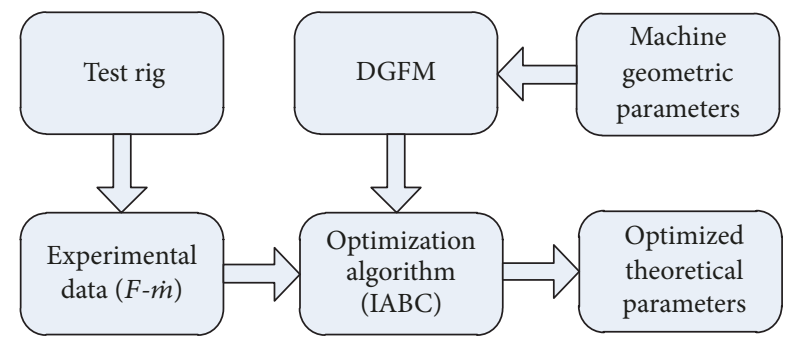

FIGURE 3: Schematic diagram of parameter estimation of DGFM.

$0.05 \%$. The sprocket rotational speed was measured by speed sensor. Frequency converter was used to change the motor speed and the sprocket rotational speed. Signal modulate circuit was necessary to bring signals to an acceptable range for recording. The data acquisition system consisted of NI USB-6216 card and computer and LABVIEW software, and the sample rate was set at $150 \mathrm{~Hz}$. All experimental data were preprocessed using EMD [30]. The theoretical parameters were estimated based on the machine geometric parameters and experimental data from test rig in the sprocket rotational speed of $320 \mathrm{r} / \mathrm{min}, 360 \mathrm{r} / \mathrm{min}$, and $400 \mathrm{r} / \mathrm{min}$, respectively. The machine geometric parameters are summarized in Table 4.

To estimate the theoretical parameters of DGFM, the population size, the maximum number of generations, and the limit values are set to 80,1000 , and 100, respectively. Figure 5 shows the $F-\dot{m}$ curve of DGFM and experimental data points, respectively. As seen from Figure 5, the $F-\dot{m}$ curves got by IABC are perfectly matched with the real data points. The accuracy of the proposed method is confirmed by this observation.

To verify the superiority of IABC algorithm in parameter estimation of DGFM, the IABC algorithm is compared with nonlinear least squares (NLS), particle swarm optimization (PSO), and genetic algorithm (GA). In all experiments in this section, the population size and the maximum number of generations used in each algorithm were chosen to be the same as the IABC algorithm, respectively. The other specific parameters of algorithms are given below.

For PSO algorithm, the learning rates $c_{1}$ and $c_{2}$ are both 2.05; the constriction factor $\chi=0.729$. For GA algorithm, a binary coded standard GA with elite units, random selection, and crossover mutation was used in the experiment. Meanwhile, single point crossover operation with the rate of 0.8 and stochastic uniform sampling technique were employed in the experiment. Mutational manipulation resumes the loss of genetic diversity during replication and hybridization applications. Mutation rate was set to be 0.01 .

Table 5 illustrates the estimated results for the theoretical parameters of DGFM when four algorithms are applied. For comparing purposes, the values of RMSE are also listed in Table 5. As it can be observed, the theoretical parameters are relatively insensitive to changes of the sprocket rotational speed. IABC and PSO result in relatively closer values of RMSE compared to NLS and GA under different sprocket rotational speed.

To make a better comparison between IABC and PSO, search range of the theoretical parameters was enlarged. The comparison results are shown in Table 6 . The value of RMSE increases obviously due to enlarging search range from Table 6; however, under different sprocket rotational speed the values of RMSE obtained by IABC algorithm are 7.62e-3, $6.99 e-3$, and $7.15 e-3$, respectively, which are still better than the results obtained by PSO $(2.36 e-2,1.58 e-2$, and $2.69 e-2)$. Figure 6 shows that the convergence rate of IABC algorithm is better than PSO algorithm at sprocket rotational speed of $360 \mathrm{r} / \mathrm{min}$. The above results show that IABC has stronger search ability for complex optimization problems and can obtain higher accuracy solutions. Therefore, IABC algorithm is better than NLS algorithm, GA algorithm, and PSO algorithm in estimating parameters of DGFM.

\section{Conclusions}

This paper presented a method of estimating parameters of DGFM using IABC algorithm. In the proposed algorithm, the research method of original $A B C$ was modified to improve its convergence rate. But the research method resulted in diversity loss, so a probability model was proposed to maintain diversity of population. With testing against a set of six benchmark functions, the IABC algorithm had a better performance than the other $\mathrm{ABC}$ variants. Then, the proposed algorithm was used to estimate parameters of DGFM. By comparing IABC algorithm with NLS algorithm, 


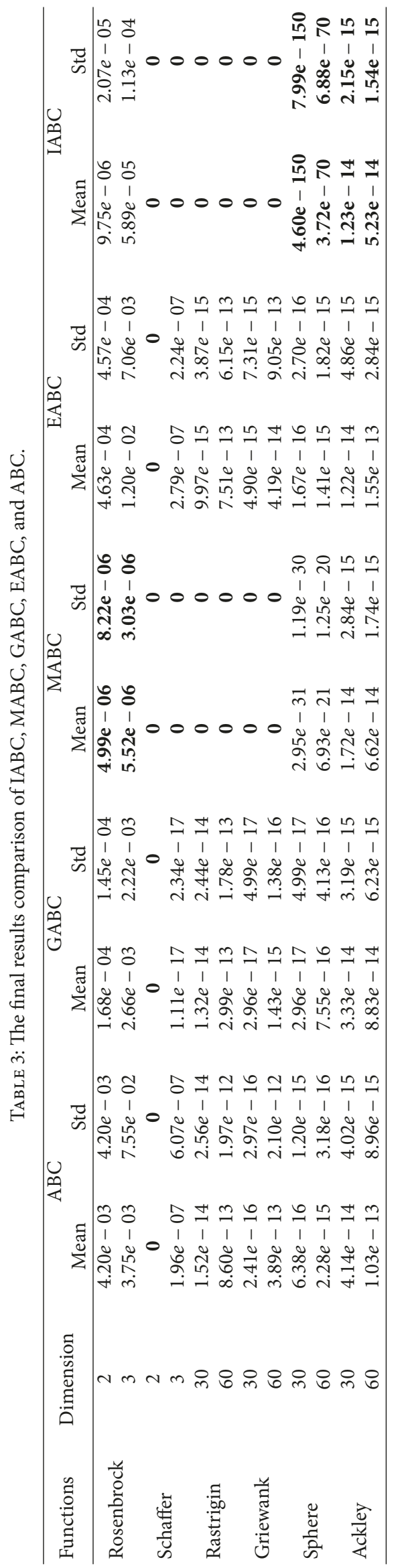


TABLE 4: Machine geometric parameters of test rig.

\begin{tabular}{lccccccc}
\hline$V(\mathrm{~m} / \mathrm{s})$ & $D(\mathrm{~m})$ & $l(\mathrm{~m})$ & $w(\mathrm{~m})$ & $d(\mathrm{~m})$ & $d_{x}(\mathrm{~m})$ & $d_{y}(\mathrm{~m})$ & $\Phi\left(^{\circ}\right)$ \\
\hline$\Omega \times w$ & 0.176 & 0.151 & 0.074 & 0.367 & 0.326 & 0.021 & 70 \\
\hline
\end{tabular}

TABLE 5: Comparison of estimated performance for NLS, PSO, GA, and IABC.

\begin{tabular}{|c|c|c|c|c|c|c|}
\hline \multirow{2}{*}{ Sprocket rotational speed $(\mathrm{r} / \mathrm{min})$} & \multirow{2}{*}{ Parameters } & \multirow{2}{*}{ Search range } & \multicolumn{4}{|c|}{ Algorithm } \\
\hline & & & NLS & PSO & GA & IABC \\
\hline \multirow{6}{*}{320} & $\mu$ & $0 \sim 1$ & 0.186 & 0.213 & 0.192 & 0.216 \\
\hline & $e$ & $0 \sim 1$ & 0.054 & 0.069 & 0.059 & 0.068 \\
\hline & $\eta$ & $750 \sim 2000$ & 1081.9 & 1123.6 & 1098.3 & 1123.3 \\
\hline & $h_{f}$ & $0 \sim 5$ & 1.984 & 2.112 & 2.031 & 2.118 \\
\hline & RMSE & & 0.013 & $9.91 e-4$ & $8.63 e-3$ & $9.76 e-4$ \\
\hline & Std & & 0.032 & $8.45 e-4$ & $6.25 e-3$ & $7.66 e-4$ \\
\hline \multirow{6}{*}{360} & $\mu$ & $0 \sim 1$ & 0.181 & 0.214 & 0.195 & 0.219 \\
\hline & $e$ & $0 \sim 1$ & 0.056 & 0.066 & 0.058 & 0.067 \\
\hline & $\eta$ & $750 \sim 2000$ & 1081.1 & 1123.8 & 1099.9 & 1123.5. \\
\hline & $h_{f}$ & $0 \sim 5$ & 1.989 & 2.114 & 2.036 & 2.119 \\
\hline & RMSE & & 0.012 & $9.36 e-4$ & $8.96 e-3$ & $9.23 e-4$ \\
\hline & Std & & 0.009 & $9.12 e-4$ & $1.38 e-2$ & $8.47 e-4$ \\
\hline \multirow{6}{*}{400} & $\mu$ & $0 \sim 1$ & 0.187 & 0.216 & 0.193 & 0.212 \\
\hline & $e$ & $0 \sim 1$ & 0.057 & 0.067 & 0.053 & 0.065 \\
\hline & $\eta$ & $750 \sim 2000$ & 1081.6 & 1123.1 & 1099.5 & 11.23 .4 \\
\hline & $h_{f}$ & $0 \sim 5$ & 1.981 & 2.111 & 2.038 & 2.117 \\
\hline & RMSE & & 0.014 & $9.41 e-4$ & $8.72 e-3$ & $9.42 e-4$ \\
\hline & Std & & 0.065 & $9.89 e-4$ & $9.65 e-3$ & $8.12 e-4$ \\
\hline
\end{tabular}

TABLE 6: Comparison of estimated performance for IABC and PSO.

\begin{tabular}{|c|c|c|c|c|c|}
\hline \multirow{2}{*}{ Algorithm } & \multirow{2}{*}{ Parameters } & \multirow{2}{*}{ Search range } & \multicolumn{3}{|c|}{ Sprocket rotational speed $(\mathrm{r} / \mathrm{min})$} \\
\hline & & & 320 & 360 & 400 \\
\hline \multirow{6}{*}{$\mathrm{IABC}$} & $\mu$ & $-1 \sim 2$ & 0.198 & 0.197 & 0.193 \\
\hline & $e$ & $-1 \sim 2$ & 0.061 & 0.069 & 0.065 \\
\hline & $\eta$ & $100 \sim 3000$ & 1118.6 & 1118.6 & 1119.3 \\
\hline & $h_{f}$ & $-5 \sim 10$ & 1.921 & 1.929 & 1.932 \\
\hline & RMSE & & $6.62 e-3$ & $5.61 e-3$ & $7.15 e-3$ \\
\hline & Std & & $4.56 e-3$ & $4.26 e-3$ & $6.42 e-3$ \\
\hline \multirow{6}{*}{ PSO } & $\mu$ & $-1 \sim 2$ & 0.179 & 0.181 & 0.186 \\
\hline & $e$ & $-1 \sim 2$ & 0.056 & 0.056 & 0.058 \\
\hline & $\eta$ & $100 \sim 3000$ & 1095.6 & 1092.8 & 1094.7 \\
\hline & $h_{f}$ & $-5 \sim 10$ & 1.881 & 1.893 & 1.863 \\
\hline & RMSE & & $3.36 e-2$ & $3.48 e-2$ & $3.69 e-2$ \\
\hline & Std & & $5.63 e-2$ & $3.12 e-2$ & $2.53 e-2$ \\
\hline
\end{tabular}




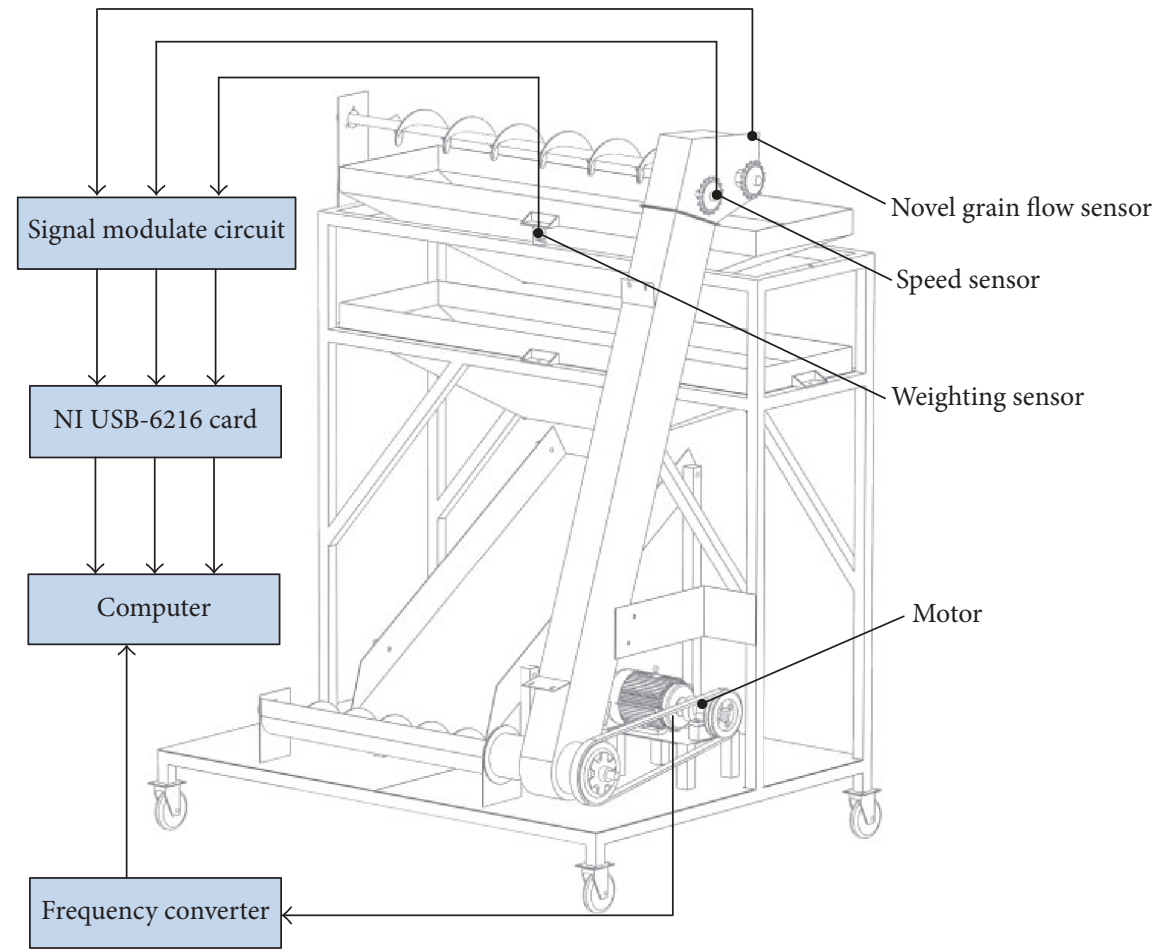

FIGURE 4: Schematic of test rig for DGFM.

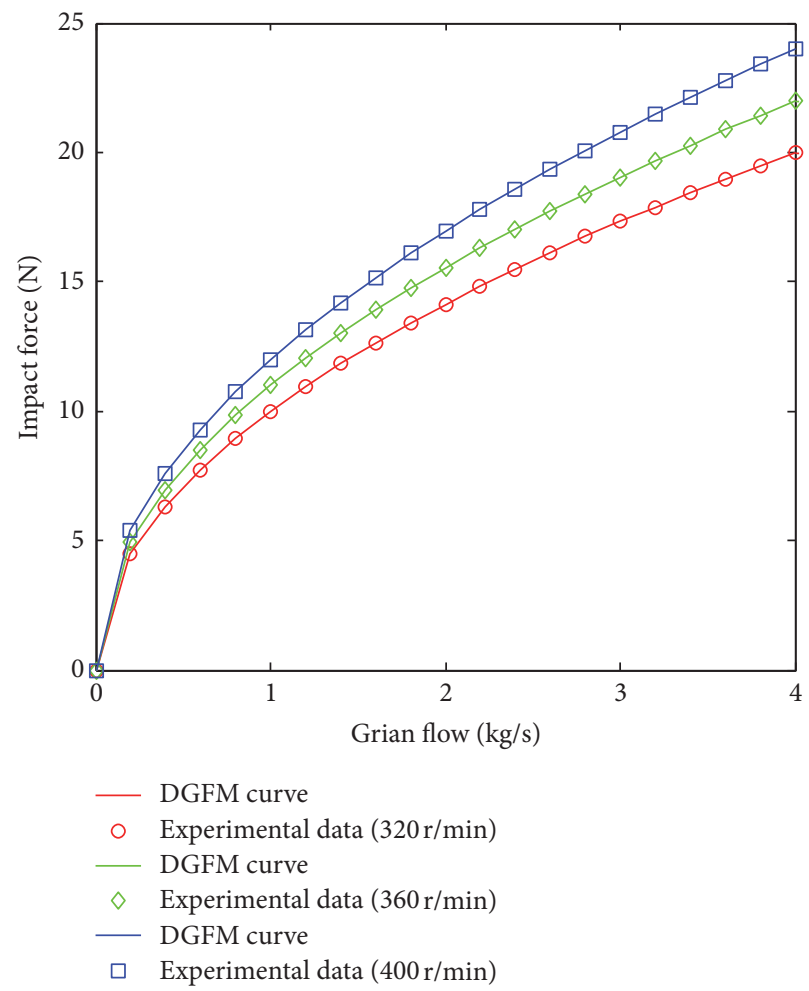

FIGURE 5: $F-\dot{m}$ curve of DGFM (using IABC algorithm) and the experimental data points. 


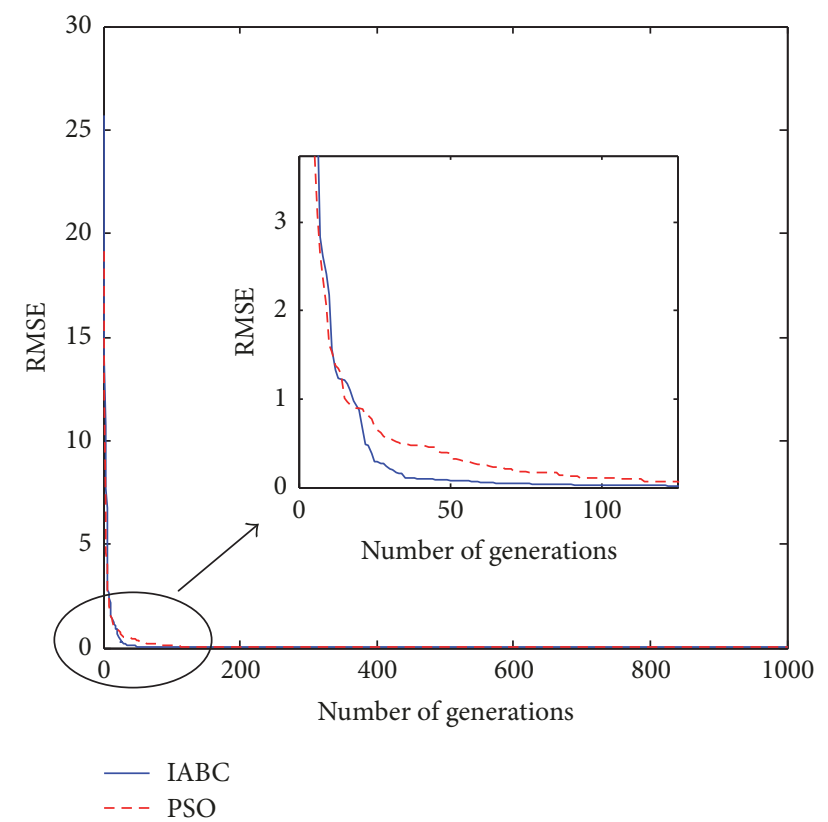

FIGURE 6: Comparison of convergence curves of IABC and PSO at sprocket rotational speed of $360 \mathrm{r} / \mathrm{min}$.

GA algorithm, and PSO algorithm, the proposed algorithm had higher accuracy solutions and a faster convergence rate, which is more suitable for parameter estimation of DGFM.

\section{Conflicts of Interest}

The authors declare that there are no conflicts of interest regarding the publication of this paper.

\section{Acknowledgments}

This project is fully supported by the Scientific Research Foundation of the Education Department of Liaoning Province, China (no. 2017LNQN22); the Young Teachers Foundation of University of Science and Technology Liaoning, China (no. 2017QN04); and the Nature Science Foundation of Liaoning Province, China (no. 2015020128).

\section{References}

[1] Z. Xin, Y. Ge, W. Bo, Y. Zhang, Y. Li, and J. Yang, "Design of microcontroller-based battery management system for pure electric vehicle," Transactions of the Chinese Society of Agricultural Engineering, vol. 30, no. 12, pp. 163-170, 2014 (Chinese).

[2] S. Arslan, F. Inanc, J. N. Gray, and T. S. Colvin, "Grain flow measurements with X-ray techniques," Computers and Electronics in Agriculture, vol. 26, no. 1, pp. 65-80, 2000.

[3] Y. W. Wang, "Monitoring the grain flow on combines device and performance study," Transactions of the Chinese Society for Agricultural Machinery, vol. 24, no. 2, pp. 42-48, 1993.

[4] X. Zhang, X. Hu, A. Zhang, Y. Zhang, and Y. Yuan, "Method of measuring grain-flow of combine harvester based on weighing," Nongye Gongcheng Xuebao, vol. 26, no. 3, pp. 125-129, 2010.
[5] M. Loghavi, R. Ehsani, and R. Reeder, "Development of a portable grain mass flow sensor test rig," Computers and Electronics in Agriculture, vol. 61, no. 2, pp. 160-168, 2008.

[6] Y. T. Li, S. Y. Liu, X. L. Zhao, H. Chen, and D. Y. Wang, "Effect of straw-bentonite-polyacrylamide composites on nitrogen adsorption of sandy soil," Transactions of the Chinese Society of Agricultural Engineering, vol. 28, no. 7, pp. 111-116, 2012.

[7] B. Qiu, G. Jiang, N. Yang, X. Guan, J. Xie, and Y. Li, "Discrete element method analysis of impact action between rice particles and impact-board," Nongye Gongcheng Xuebao, vol. 28, no. 3, pp. 44-49, 2012.

[8] N. Hemming and J. Chaplin, "Precision of real time grain yield data," in Proceedings of the ASAE Annual International Meeting 2004, pp. 747-756, can, August 2004.

[9] M. D. Schrock, D. L. Oard, R. K. Taylor et al., "Diaphragm impact sensor for measuring combine grain flow," Applied Engineering in Agriculture, vol. 15, no. 6, pp. 639-642, 1999.

[10] X. H. Wei, J. M. Zhang, Z. M. Dan, and C. L. Liu, "Signal processing method of impact-based grain flow sensor for predicted yield," Transactions of the Chinese Society of Agricultural Engineering, vol. 30, no. 15, pp. 222-228, 2014 (Chinese).

[11] K. Shoji, I. Matsumoto, and T. Kawamura, "Impact-by-impact sensing of grain flow on jidatsu combine," Engineering in Agriculture, Environment and Food, vol. 4, no. 1, pp. 1-6, 2011.

[12] J. Anthonis, G. Strubbe, K. Maertens, J. De Baerdemaeker, and H. Ramon, "Design of a friction independent mass flow sensor by force measurement on a circular chute," Biosystems Engineering, vol. 84, no. 2, pp. 127-136, 2003.

[13] D. Hennens, J. Baert, B. Broos, H. Ramon, and J. De Baerdemaeker, "Development of a flow model for the design of a momentum type beet mass flow sensor," Biosystems Engineering, vol. 85, no. 4, pp. 425-436, 2003.

[14] J. Zhou, B. Cong, and C. Liu, "Elimination of vibration noise from an impact-type grain mass flow sensor," Precision Agriculture, vol. 15, no. 6, pp. 627-638, 2014.

[15] K. Shoji, H. Itoh, and T. Kawamura, "In-situ non-linear calibration of grain-yield sensor: Optimization of parameters for flow rate of grain vs. Force on the sensor," Engineering in Agriculture, Environment and Food, vol. 2, no. 3, pp. 78-82, 2009.

[16] "The effects of parameters settings of ant colony algorithm on the performance of ultrasonic echo estimation," SCIENCE CHINA Information Sciences.

[17] M.-W. He, L.-L. Sun, K.-Y. Hu, Y.-L. Zhu, and H.-N. Chen, "Analysis of DoD inkjet printhead performance for printable electronics fabrication using dynamic lumped element modeling and swarm intelligence based optimal prediction," Journal of Central South University, vol. 22, no. 10, pp. 3925-3934, 2015.

[18] J. J. Soon and K. Low, "Photovoltaic model identification using particle swarm optimization with inverse barrier constraint," IEEE Transactions on Power Electronics, vol. 27, no. 9, pp. 39753983, 2012.

[19] A. Montazeri, C. West, S. D. Monk, and C. J. Taylor, "Dynamic modelling and parameter estimation of a hydraulic robot manipulator using a multi-objective genetic algorithm," International Journal of Control, vol. 90, no. 4, pp. 661-683, 2017.

[20] P. Mukherjee and L. Satish, "Construction of equivalent circuit of a single and isolated transformer winding from FRA data using the $\mathrm{ABC}$ algorithm," IEEE Transactions on Power Delivery, vol. 27, no. 2, pp. 963-970, 2012.

[21] A. Şencan Şahin, B. Kiliç, and U. Kiliç, "Design and economic optimization of shell and tube heat exchangers using Artificial 
Bee Colony (ABC) algorithm," Energy Conversion and Management, vol. 52, no. 11, pp. 3356-3362, 2011.

[22] K. Ayan and U. Kilıç, "Artificial bee colony algorithm solution for optimal reactive power flow," Applied Soft Computing, vol. 12, no. 5, pp. 1477-1482, 2012.

[23] R. Reinke, H. Dankowicz, J. Phelan, and W. Kang, "A dynamic grain flow model for a mass flow yield sensor on a combine," Precision Agriculture, vol. 12, no. 5, pp. 732-749, 2011.

[24] D. Karaboga and B. Basturk, "On the performance of artificial bee colony (ABC) algorithm," Applied Soft Computing, vol. 8, no. 1, pp. 687-697, 2008.

[25] G. Zhu and S. Kwong, "Gbest-guided artificial bee colony algorithm for numerical function optimization," Applied Mathematics and Computation, vol. 217, no. 7, pp. 3166-3173, 2010.

[26] W. Gao, S. Liu, and L. Huang, "A global best artificial bee colony algorithm for global optimization," Journal of Computational and Applied Mathematics, vol. 236, no. 11, pp. 2741-2753, 2012.

[27] E. Mezura-Montes and R. E. Velez-Koeppel, "Elitist Artificial Bee Colony for constrained real-parameter optimization," in Proceedings of the 2010 6th IEEE World Congress on Computational Intelligence, WCCI 2010 - 2010 IEEE Congress on Evolutionary Computation, CEC 2010, Spain, July 2010.

[28] H. Wang, J. T. Hu, L. Gao, and Y. F. Jia, “Development and optimization of a novel grain flow sensor based on PVDF piezoelectric film," vol. 9, pp. 143-152, 2016.

[29] H. Wang, X. P. Bai, and H. B. Liang, "Proportional distribution method for estimating actual grain flow under combine harvester dynamics," International Journal of Agricultural and Biological Engineering, vol. 10, no. 4, pp. 158-164, 2017.

[30] H. Wang and J. Hu, "Grain flow signal reduction noise using EMD," The International Agricultural Engineering Journal, vol. 24, no. 2, pp. 152-158, 2015. 


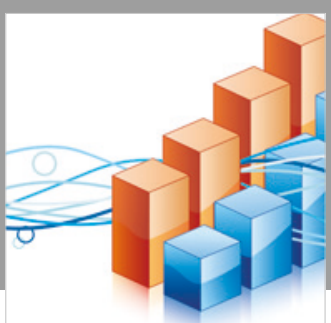

Advances in

Operations Research

\section{-n-m}
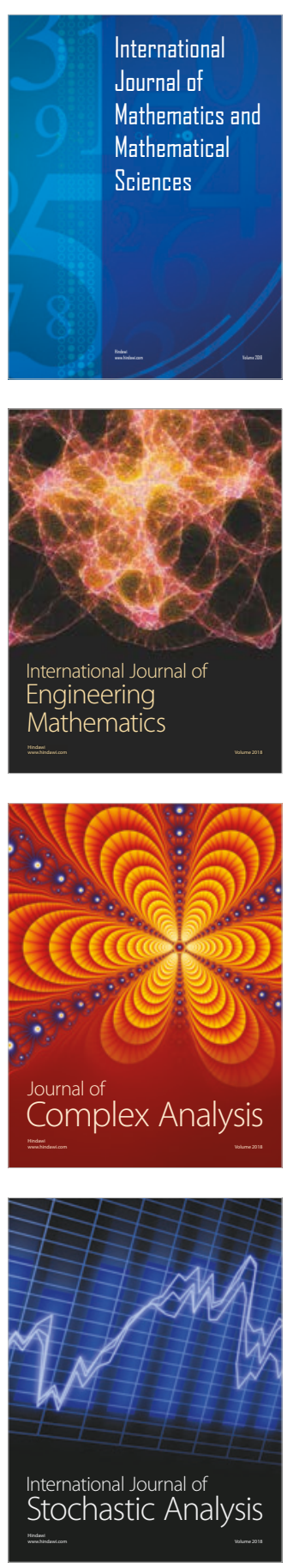
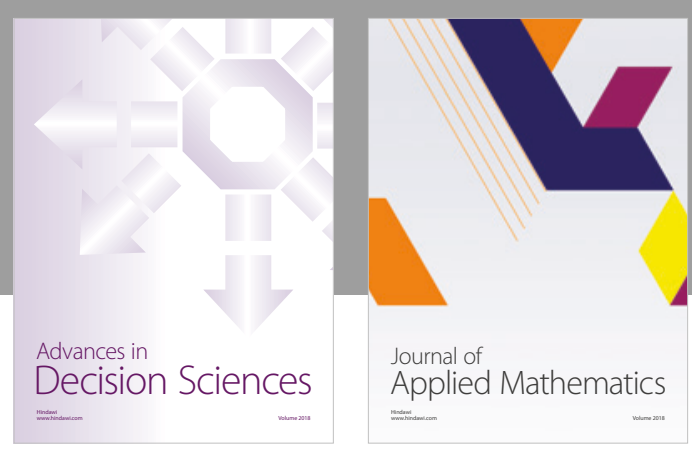

Journal of

Applied Mathematics
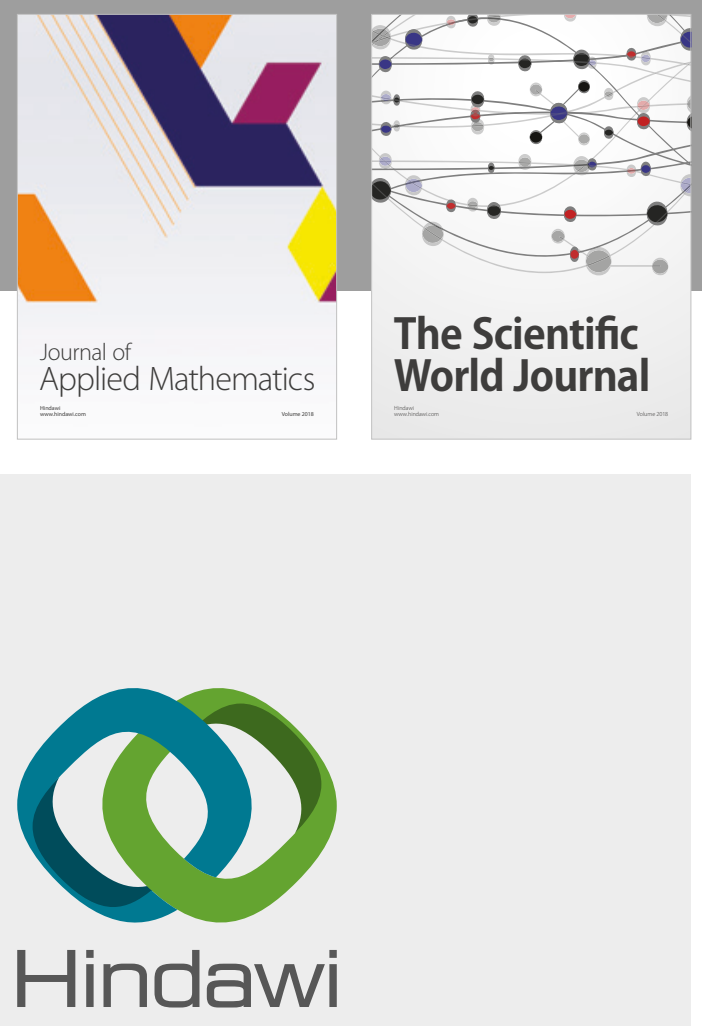

Submit your manuscripts at

www.hindawi.com

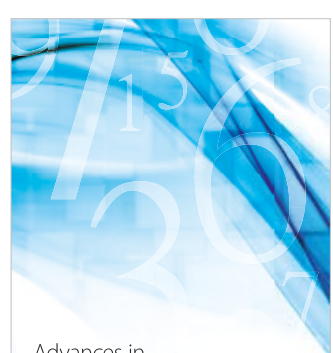

Advances in
Numerical Analysis
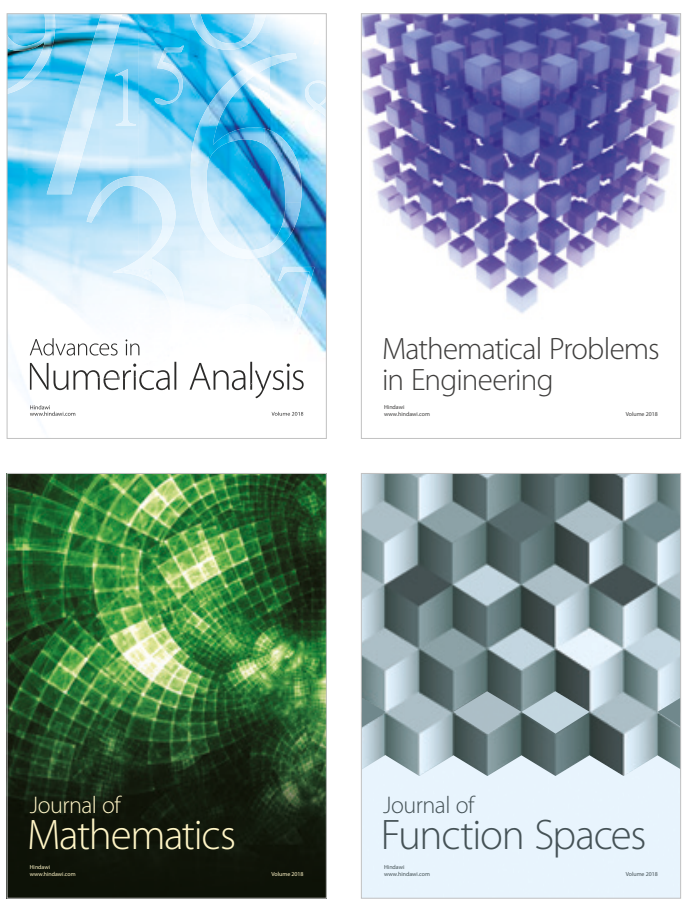

Mathematical Problems in Engineering

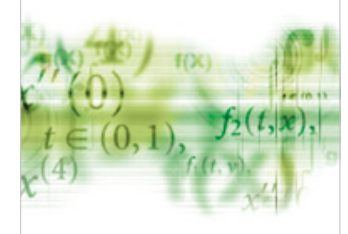

International Journal of

Differential Equations

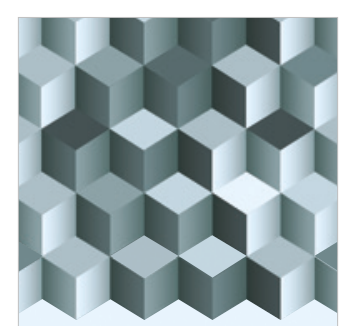

Journal of

Function Spaces

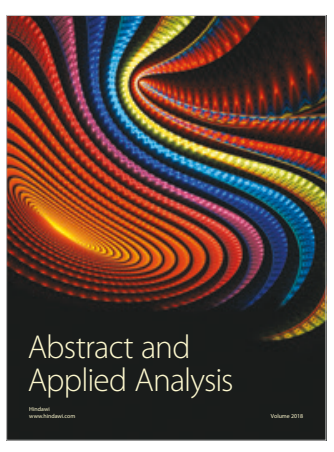

The Scientific

World Journal

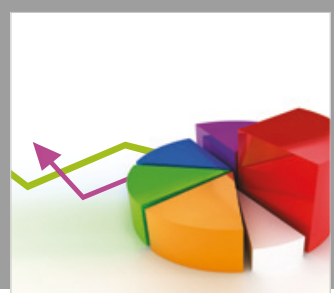

Journal of

Probability and Statistics
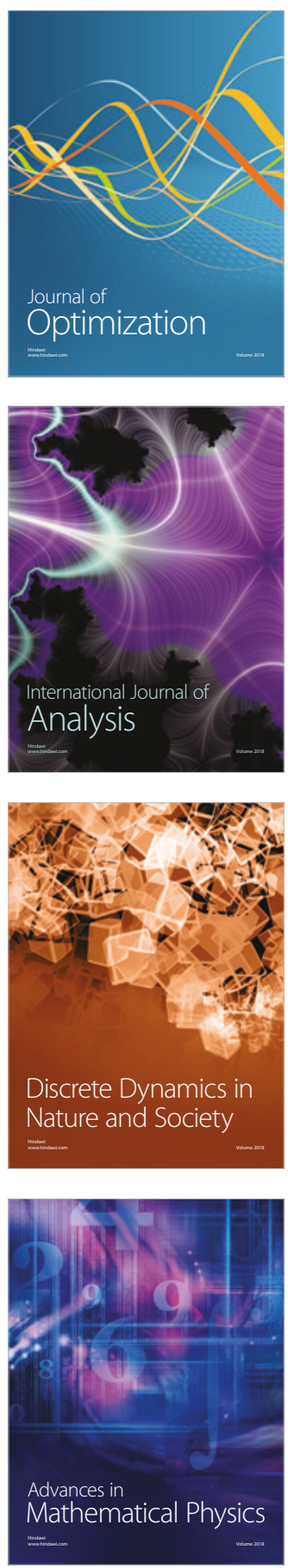